\title{
Prólogo \\ Buen Vivir: ¿Una auténtica alternativa post-capitalista?
}

\author{
Fernando de la Cuadra
}

fmdelacuadra@gmail.com

La concepción del Buen Vivir se propone desnudar y superar los errores y las limitaciones de la matriz de pensamiento eurocentrista, de una determinada narrativa de la modernidad y del capitalismo como única forma posible de pensar y vivir. Ello se encuentra asociado a las diversas nociones y teorías tradicionales del progreso y el desarrollo que se sustentan en el crecimiento exponencial de bienes y servicios lo cual supone la explotación ilimitada de los recursos naturales y humanos que existen en el planeta. Para alcanzar los beneficios que presume la distribución de los frutos de este crecimiento económico persistente, se elaboran planes, programas y planes de desarrollo, proceso reforzado por un conjunto de instancias financieras, de capacitación y transferencia de conocimientos desde el mundo desarrollado hacia el mundo en vías de desarrollo. Esta especie de mandato sacrosanto se transformó en una verdad única e incuestionable que acabó por someter o ignorar toda y cualquier perspectiva surgida fuera del canon occidental de formación de la modernidad y del capitalismo como proyecto civilizatorio.

La abundante información elaborada hasta ahora por los científicos dejaría meridianamente claro que dicho paradigma está destinado al fracaso. En efecto, el presente modelo ha generado un crecimiento exponencial en la explotación de los recursos naturales y ha estimulado un consumismo desenfrenado, especialmente en los países del hemisferio norte. Por lo mismo, es responsable tanto de provocar un agotamiento de los recursos como de producir toneladas de basura que contaminan diariamente las aguas, el aire y la tierra. ${ }^{1}$ Cada año se pierden 14,6 millones de hectáreas de bosques y miles de especies, reduciendo y erosionando irreversiblemente la diversidad biológica. Continúa la devastación de las selvas, con lo cual el mundo pierde anualmente cerca de 17 millones de hectáreas, que equivalen a cuatro veces la extensión de Suiza. Y como no hay árboles que absorban los excedentes de CO2, el efecto invernadero y el recalentamiento se agravan. La información generada por los científicos del Panel Intergubernamental sobre Cambio Climático (IPCC) señala que necesitamos reducir el nivel de equivalentes de CO2 en la atmósfera para 350 partes por millón. Actualmente este nivel se encuentra en 390 partes por millón y la tendencia es que continúe en aumento. El dióxido de carbono presente en la atmósfera se ha incrementado en un 32\% respecto del siglo XIX, alcanzando las mayores concentraciones de los últimos 20 millones de años, y se calcula que estas emisiones se acrecienten un 75\% entre 1997 y 2020. La capa de ozono, a 
pesar del Protocolo de Montreal, no se recuperará hasta mediados del siglo XXI. Cada año emitimos cerca de 100 millones de toneladas de dióxido de azufre, 70 millones de óxidos de nitrógeno, 200 millones de monóxido de carbono y 60 millones de partículas en suspensión, agravando los problemas causados por las lluvias ácidas, el ozono troposférico y la contaminación atmosférica local.

En definitiva, un conjunto de indicadores medioambientales estudiados en las últimas décadas parecen revelar cada vez con mayor claridad que si la humanidad no cambia su estilo de desarrollo, en menos de un siglo colocaremos en serio riesgo la supervivencia del planeta y del género humano. Muchas de las soluciones que se han elaborado para contornar los efectos perversos del modelo productivista que impera en el mundo ha sido la generación de mecanismos de mercados para limitar la emisión de contaminantes, como el sistema de mercado de carbono. Este tipo de respuestas solo vienen a legitimar la acción de las empresas y naciones contaminadoras que compran en la bolsa "verde" su licencia para seguir contaminando. Como nos recuerda Mészáros, a cada nueva fase de postergación forzada, las contradicciones del sistema del capital sólo se pueden agravar, acarreando consigo un peligro aún mayor para nuestra propia sobrevivencia.

Contrariamente, el Buen Vivir cuestiona la esencia de este padrón productivista y consumista que viene organizando el planeta a partir de una perspectiva evolucionista, lineal que supone que todas las sociedades deben transitar desde un ámbito atrasado, tradicional o subdesarrollado hacia una etapa superior identificada con la modernidad, la industrialización y el progreso. El Buen Vivir nos advierte sobre la inviabilidad de continuar manteniendo el actual esquema de producción y consumo, concebido como un dispositivo legítimo de crecimiento basado en la acumulación permanente de bienes materiales. Para el Buen Vivir, diferentemente, la riqueza no consiste en tener y acumular la mayor cantidad de bienes posibles, sino en lograr un equilibrio entre las necesidades fundamentales de la humanidad y los recursos disponibles para satisfacerlas.

Sumak Kawsay en Quechua, Suma Qamaña en Aymara o Buen Vivir en la traducción más difundida, representa una cosmovisión construida a través de muchos años por los pueblos altiplánicos de los Andes. Ella no encarna necesariamente una manera de pensar y actuar de las comunidades altiplánicas, pues dicha perspectiva también es parte de la vida de otros pueblos originarios, como las comunidades Mapuche del sur, las poblaciones que habitan en la región amazónica o los diversos grupos autóctonos diseminados por todo el continente. De esta forma, el Buen Vivir se ha constituido en una propuesta y en una oportunidad para pensar otra realidad en las cual los seres humanos forman parte de un todo más armónico con la naturaleza y con los otros humanos, con la alteridad que nos enriquece cotidianamente. Es el reconocimiento de que existen diversos valores y formas de concebir el mundo, de respeto por todos los seres vivos que integran y conviven en nuestra casa común, la tierra. Su visión utópica se 
ha venido complementando y ampliando a través de la incorporación de múltiples discursos y saberes diseminados por los más diferentes rincones del planeta.

Por lo tanto, el Buen Vivir no es patrimonio de ningún grupo o sector social en particular, ni tampoco supone una fórmula mágica o catecismo al cual hay que adherir religiosamente. Es una propuesta en construcción permanente, es una concepción que parte de la idea de que existe una diversidad cultural, una pluralidad que se enriquece permanentemente en la convivencia cotidiana y que encuentra su armonía precisamente en el reconocimiento de esas diferentes formas de vivir. Es la búsqueda de una vida en fraternidad y cooperación del ser humano consigo mismo, con sus pares y con el conjunto de los seres que habitan en la naturaleza, todos formando parte de una entidad indisoluble e interdependiente, cuya existencia se delimita a partir de los otros. Tal visión no implica por cierto desconocer que en la sociedades coexisten las diferencias sociales, los conflictos y las desavenencias entre sus miembros. Lo que el Buen Vivir plantea es que se puedan superar estos obstáculos y desacuerdos en torno a una consciencia y un compromiso colectivo que permita cimentar una vida más plena y sustentable para todos.

En América Latina la emergencia de gobiernos progresistas auguraba la expectativa de que las nociones tradicionales de desarrollo y progreso, asociadas a la idea de crecimiento, fueran modificadas o expurgadas del vocabulario coloquial. Sin embargo, ello no ha ocurrido y podemos observar que la mayor parte de los gobiernos llamados de "progresistas" continúan cautivos a una visión tradicional del desarrollo, afectando con sus actividades (públicas y privadas) a pueblos originarios y comunidades campesinas existentes en la región. Por lo mismo se torna pertinente pensar en valores que han sido parte del arsenal de la humanidad, pero que han sido postergados en función de las fuerzas económicas y mercantiles. El Buen Vivir sintetiza esta alternativa o la posibilidad de pensar otro tipo de modelo. No obstante lo anterior, es necesario consignar que la noción de Buen Vivir es lo suficientemente amplia y abstracta -y por lo mismo muchas veces ambigua- que impide o limita su aplicación como política pública.

En el caso de Bolivia o Ecuador, países que han incorporado en sus constituciones los derechos de la Naturaleza, las medidas concretas hacia el Buen Vivir se han visto bloqueadas por las diferentes concepciones sobre las formas más adecuadas de operacionalizar o poner en práctica una agenda del Buen Vivir a través de las acciones gubernamentales. En efecto, el gobierno de Evo Morales se ha dedicado preferencialmente a desarrollar una política social intensa, de alfabetización, distribución de renta, participación del Estado en la producción de gas y petróleo, incentivo a la mediana y pequeña empresa, pero manteniendo una relación contradictoria y tensionada con las comunidades originarios y con la naturaleza. El caso TIPNIS quizás sea el ejemplo más expresivo de esta modalidad discordante para llevar adelante los principios del Buen Vivir. 


\section{Articulación con otras propuestas alternativas}

Considerando los problemas y límites impuestos por los modelos clásicos de desarrollo, han surgido diversas corrientes de pensamiento o paradigmas que pretenden erigirse como alternativas al padrón dominante. Muchas de estas iniciativas han sido impulsadas por una variedad de grupos relativamente pequeños, pero que en su globalidad representan un porcentaje significativo de la población mundial. Precisamente desde una crítica vehemente a la noción clásica de desarrollo, un grupo de autores (Escobar, Shiva, Esteva, Rahnema y Bawtree, entre otros) han propuesto una perspectiva que pretende superar las limitaciones y ampliar los horizontes de dicha concepción. Partiendo de la constatación de que este concepto fue cimentado históricamente desde la exclusión de las diversas voces y saberes, el llamado postdesarrollo se constituye como un modelo que parte de la valorización de las culturas vernáculas y de la idea de depender menos del conocimiento de los expertos y más del conocimiento generado por las personas que aspiran a construir un mundo más humano y sostenible en términos culturales y ecológicos. Ello implica la necesidad de cambiar las prácticas del "saber" y del "hacer” que definen el actual régimen de desarrollo y, por lo tanto, multiplicar los centros y agentes de producción del conocimiento. Lo anterior supone visibilizar aquellas formas de conocimiento que son generadas por quienes supuestamente son los objetos del desarrollo para que puedan transformarse en sujetos y agentes. ${ }^{2}$

El postdesarrollo concibe también que las personas y las comunidades no están necesariamente abocadas a satisfacer sus necesidades materiales, pues ellas forman parte de una constelación más amplia, pero acotada, de necesidades construidas culturalmente. Anteriormente, una crítica a la afirmación del carácter infinito de las necesidades ya había sido realizada por la vertiente del desarrollo a escala humana (Max-Neef, Elizalde y Hopenhayn). En efecto, los defensores del desarrollo a escala humana plantean que a diferencia de lo que generalmente se piensa, las necesidades humanas son finitas y se encuentran en permanente interacción. Ellas pueden ser pueden ser definidas y clasificadas de acuerdo con dos criterios: existencial y axiológico. ${ }^{3}$ En síntesis, dicha concepción sostiene la idea de que el desarrollo se debe concentran en constituir un conjunto de satisfactores adecuados para atender las necesidades humanas fundamentales que permitan la generación de niveles crecientes de interdependencia entre los seres humanos, entre ellos mismos y en su articulación con la naturaleza, en la interacción de los procesos globales y los comportamientos a escala local y en la imbricación del ámbito personal con su entorno social.

En dialogo fecunda con esta concepción en que la humanidad puede efectivamente alcanzar a satisfacer sus necesidades dentro de un umbral sustentable y por medio de mecanismos que no pasan obligatoriamente por los mercados capitalistas, se encuentra toda la tradición derivada de aquello que fue llamado por Marcel Mauss como el “espíritu del don”.Esta pers- 
pectiva pretende dar cuenta de un tipo de relación que se establece entre los hombres en la cual la reciprocidad desempeña un papel fundamental, contrariamente a los intercambios de equivalentes realizados simultáneamente que se producen en la economía de mercado capitalista. A partir de la noción de los tres momentos de la reciprocidad expuestos por Marcel Mauss en sus estudios sobre las comunidades aborígenes (dar - recibir - retribuir), un conjunto de autores (Boilleau, Caillé, Godbout, Insel, Kolm, entre otros) viene rediscutiendo la función del mercado, el valor de cambio, el interés, la impersonalidad y el utilitarismo como iconos incontestados de la sociedad moderna. Estos autores descubrieron que el espíritu de don o dádiva posee una fuerza indiscutible entre las personas, fuerza que permite establecer y consolidar los lazos existentes en la comunidad y en las sociedades contemporáneas. Por ejemplo, autores principalmente franceses vinculados al grupo M.A.U.S.S. (Mouvement anti-utilitariste dans les sciences sociales) demostraron que los circuitos de reciprocidad no se producen solamente en la sociedades tribales estudiadas por el propio Mauss, sino que tales circuitos de trocas reciprocas están íntimamente presentes y actuantes en nuestras sociedades.Prácticamente todos los sistemas de voluntariado, cuidados de enfermos, donación de sangre y de órganos, trabajos por el bien de la vecindad, etc. se basan en comportamientos de generosidad con los extraños, acciones derivadas de gestos de buena voluntad, desprendimiento y libertad del donante. Esta dimensión de la actividad humana representa para estos autores, una forma de reconstruir y consolidar el tejido o lazo social existente entre las personas. ${ }^{4}$ Estas nuevas formas de reciprocidad constituyen, por lo tanto, un tipo de contrato de la civilidad, que no es más el contrato político con el Estado, sino un contrato de cada persona con todos aquellos que forman parte de la colectividad. El espíritu del don - a diferencia de los intercambios de mercado- crea una relación, un vínculo entre los actores de dicho intercambio, el cual no tiene un límite de tiempo demarcado. Aquí, los bienes que participan en la permuta poseen principalmente un valor simbólico, valor de uso marcado por las relaciones que surgen y se establecen en función de ese bien.

Una perspectiva que también privilegia una relación ponderada entre las necesidades humanas, los bienes de consumo y una producción delimitada para satisfacer estas necesidades fundamentales ha recibido el nombre de decrecimiento. ${ }^{5}$ Tal como lo advierte uno de sus principales propulsores, la palabra decrecimiento posee más que nada una fuerza propagandística, es un slogan político que posee implicaciones teóricas:

“La palabra de orden ‘decrecimiento’ tiene como principal meta enfatizar fuertemente el abandono del objetivo del crecimiento ilimitado, objetivo cuyo motor no es otro sino la búsqueda del lucro por parte de los detentores del capital, con consecuencias desastrosas para el medio ambiente y por tanto para la humanidad. No solo la sociedad queda condenada a no ser más que el instrumento o el medio de la mecánica productiva, sino que el propio hombre tiende a transformarse en la víctima de un sistema que va a transformarlo en un inútil y prescindir de él”. (Latouche, 2009, pp. 4-5). 
El decrecimiento es una opción de desarrollo diferentemente de los presupuestos del modelo productivista, es una perspectiva que nació para enfrentarse a aquellas visiones del desarrollo sostenible que era y continúa siendo enarbolada por las empresas y que quieren convertir el llamado desarrollo verde o ecológico en una nueva oportunidad de negocios. Es un proyecto global y a la vez revolucionario, pues implica un cambio a largo plazo en que las empresas y los consumidores estén dispuestos a mudar el patrón predatorio y de consumo existente hasta ahora, su objetivo es lograr que la sociedad se autolimite para conseguir el bienestar de todos. Supone poner en marcha una reorganización de nuestras vidas, la producción, el transporte y el consumo a través de formas más conscientes de consumo y por medio de una vida más simple, sin grandes parafernalias que nos rodeen, utilizando estrictamente lo que necesitamos para llevar una vida digna y plena. Esta visión se vincula con el movimiento por una opción de simplicidad voluntario y un estilo de vida leve, más liviana, más lenta, como aquel levantado por el también movimiento slow food. Una tendencia también vinculada a la anterior es aquella inaugurada con la publicación del libro Elogio de la lentitud de Carl Honoré ${ }^{6}$ que como lo dice el subtítulo, se ha transformado en una especie de biblia que desafía el culto a la vorágine contemporánea en que estamos todos envueltos.

La idea del decrecimiento ha sido considerada ilusa y atacada desde diversos ángulos. En primer lugar, porque el mundo necesita seguir creciendo para alimentar a sus habitantes. Pero el decrecimiento no implica necesariamente dejar de producir, sino que producir a una escala moderada. Y de hecho las recientes evidencias sobre el calentamiento global y cambio climático que aquejan al planeta apuntan en otra dirección; la alternativa por el decrecimiento y la discusión sobre el poder y la desigual distribución del uso de los recursos naturales es ciertamente parte imprescindible de cualquier agenda que pretenda discutir el futuro de la humanidad. En ese sentido, el debate sobre el decrecimiento puede ser considerado un elemento fundamental para pensar en la construcción de un proyecto ecologista y socialista, puesto que incluye en su seno la concepción de que es preciso avanzar hacia una modalidad diferente de funcionamiento de la sociedad, más democrática, más igualitaria y más incluyente que redefina drásticamente el actual modelo de producción y consumo, intentando alcanzar el bienestar de todos en el marco de un nuevo relacionamiento de la humanidad entre ella misma y con la naturaleza.

De esta manera, estrechamente vinculado y dialogando con el proyecto por el decrecimiento se encuentra toda la tradición por un socialismo ecológico, tendencia inaugurada por William Morris en el siglo XIX. Este socialismo ecológico o eco-socialismo representa también una reorganización de la vida en muchos ámbitos, supone pensar en el uso de energías alternativas y limpias, supone reducir la huella ecológica a través de actividades en escala local y de relaciones más equitativas entre los miembros de la comunidad. De esta manera, el ecosocialismo busca romper drásticamente con las prácticas destructivas y las formas predadoras que derivan de un modo de producción y consumo altamente demandante de recursos natura- 
les y humanos. La respuesta ecosocialista representa una ruptura tanto con el modelo expansionista del capital como con la perspectiva productivista del “socialismo real”. Para los ecosocialistas, ya sea la lógica del mercado y del lucro o ya sea el productivismo burocrático del marxismo economicista y vulgar, son considerados modelos totalmente incompatibles con la urgente e impostergable exigencia de preservar y cuidar del medioambiente y las personas. ${ }^{7}$

Así, la propuesta del socialismo ecológico y la perspectiva del decrecimiento representan una reorganización de la vida en muchos ámbitos, suponen renunciar al consumo artificial para emprender un consumo autolimitado y adecuado a las necesidades reales de las personas, lo cual supone pensar también en el uso de energías alternativas y limpias y recudir la huella ecológica a través de actividades en escala local y de relaciones más equitativas y armónicas entre los miembros de la comunidad.

En esta búsqueda de una vida más relajada, más consciente, otras iniciativas han surgido en este último periodo o muy recientemente. Por ejemplo, en la comunidad Avaaz es un grupo de acción que busca a través de la adhesión de numerosas firmas de peticiones de miembros en todo el mundo, influir sobre diversos aspectos de la vida en el planeta, desde la elaboración de políticas públicas hasta el financiamiento de acciones en pro de determinadas pueblos y comunidades. Una de las últimas campañas de este grupo consiste en la difusión de una forma de cambiar el mundo por medio de la "jornada interior". Esta jornada implica asumir tres principios fundamentales, a saber:

Mostrar gentileza y respeto: Seremos gentiles e tendremos respeto con nosotros mismos y con los otros siempre que sea posible. Y siempre será posible, pues cada persona que encontramos en la vida puede estar pasando por una batalla de la cual nada sabemos.

Buscar la sabiduría: Vamos a aspirar a más sabiduría en nuestras decisiones, escuchar profundamente a nosotros mismos y a los otros, y buscar el equilibrio entre razón, corazón e intuición en armonía con lo que creemos es lo correcto.

Practicar la gratitud: Vamos constantemente a reflexionar sobre las cosas por las cuales debemos agradecer; eso nos entrega perspectiva, diluye la negatividad y nos hace centrarnos en lo que realmente importa.

A partir de estas breves consideraciones surgieron algunas interrogantes esenciales: ¿El Buen Vivir representa un nuevo paradigma para reencontrar este equilibrio? o ¿representa una alternativa factible para concebir una sociedad post-capitalista? O también cabe cuestionarse si ¿El Buen Vivir puede convertirse en una revolución cultural que, en el marco del capitalismo, reduzca las consecuencias perversas del individualismo, el hedonismo extremo y el consumo desatado? ¿Existe el riesgo de que el Buen Vivir no pase de ser una moda conceptual que con el transcurso del 
tiempo se vaya diluyendo hasta convertirse en una palabra más que se incorpora al léxico de las agencias del poder supranacional? Estas son algunas de las muchas preguntas que quisimos abrir para la reflexión en el presente número temático de Revista Polis. En ese esfuerzo, el Lente de aproximación buscó además abordar propositivamente los posibles caminos para construir una vida más armónica del ser humano consigo mismo, con sus congéneres y con el mundo natural, entendiendo que frente a los efectos nocivos del crecimiento ilimitado que impera actualmente es ineludible pensar y poner en práctica una nueva forma de vida más digna y sostenible para el conjunto de los habitantes del planeta.

Abriendo el Lente de aproximación, el artículo “La transdisciplinariedad una herramienta para apuntar al Buen Vivir” realizado porun grupo de académicos de la Universidad Nacional Autónoma de Nicaragua (UNAN) se proponeexponer la relevancia que tiene la construcción del diálogo transdisciplinar entre trece comunidades rurales vinculadas a la Unión de Campesinos Organizados de San Dionisio (UCOSD) y los profesionales asociados al Programa de Post-grado en Desarrollo Rural Territorial Sustentable de esa casa de estudios. A partir de este vínculo, surgió la demanda por parte de la organización para que estos profesionales los pudieran apoyar en el análisis de la realidad socioeconómica y productiva de las familias y comunidades, a través de un proceso participativo y transdisciplinario, que permitiera generar un conjunto de propuestas de desarrollo a ser implementadas por la organización o gestionadas ante otros actores locales.Para avanzar en este dialogo transdisciplinar se utilizó la matriz de necesidades y satisfactores expuesta por los creadores de la perspectiva de desarrollo a escala humana, debatiendo en cada una de las comunidades sus múltiples necesidades a partir de las diferentes categorías existenciales y axiológicas contenidas en dicha matriz. El resultado de esta experiencia dialógica que se estableció entre el conjunto de actores en el marco del proyecto, puede representar efectivamente una herramienta que apunte hacia el Buen Vivir.

La sección monográfica continúa con el documento “¿Qué significa Buen Vivir en los asentamientos irregularesde América Latina?” de las autoras Sabina Cárdenas y María Verónica D’Inca. Ellas se sitúan en el ámbito de la arquitectura y la planeación urbana para abordar la problemática de los asentamientos irregulares de América Latina y efectuar una revisión crítica con relación a las tres vertientes asociadas a los asentamientos irregulares que permita formular una alternativa desde la arquitectura, a saber: a) el higienismo en el que se apoya su expresión concreta, la erradicación; b) la no intervención contraria al higienismo y que defiende el modelo de autogestión; y c) la interacción situacional que supone la construcción y reconstrucción en el tiempo no solo de lo tangible sino de lo intangible, del espacio cargado de memorias y de deseos. Inspiradas en las categorías existenciales y axiológicas de las necesidades humanas (Max-Neef, Elizalde y Hopenhayn) y en la escala de la ciudad-barrio (Alguacil), las autoras diseñan una posible salida desde la arquitectura para los asentamientos irregularesque privilegiando las nociones de solidaridad, articulación, di- 
versidad, autonomía y escala humana permita construir la ciudad cuidando las especificidades de cada barrio y resaltando el papel de la esfera pública,más que la privada, en la configuración del espacio urbano.

En el tercer trabajo de esta sección titulado "Heterogeneidad estructural y socialismo del Buen Vivir”, Wolney Roberto Carvalho y Félix Pablo Friggeri se plantean como propósito subrayar la importancia de la concepción de heterogeneidad estructural existente en América latina, como un aspecto que reviste una enorme potencialidad a la hora de pensar proyectos alternativos para la región. Para ello se apoyan en un conjunto de autores (Pinto, Rangel, Furtado, Fernandes,Quijano), pero es a partir de Mariátegui y que ellos recuperan el concepto de Socialismo Indoamericano como una expresión que viene a alimentar la noción de Socialismo de Buen Vivir, expresión que para los autores, puede acercarse a una búsqueda latinoamericana de valorización del potencial de aquel mundo considerado como "atrasado" por diversos discursos y que sin embargo representaría una visión alternativa potente frente el predominio avasallador del capitalismo. Este horizonte post-capitalista se encontraría, en definitiva, enrizado en la vida y en la praxis fundamentalmente de nuestros pueblos originarios, por los movimientos campesinos y, también, por intelectuales solidarios con esta perspectiva del Buen Vivir.

Sigue la secuencia con el texto " $O$ Buen Vivir e os saberes ancestrais frente ao neo-extrativismo do século XXI” de Bruna Huertas Fuscaldo y Vivian Urquidi de la Universidad de São Paulo (USP), en el que ambas investigadoras reflexionan en torno a la contribución del concepto de Buen Vivir como una tentativa de erguir un pensamiento contra-hegemónico al paradigma civilizatorio de la modernidad eurocéntrica colonialista y capitalista que impera en nuestros días. En ese contexto, el trabajo recoge algunos aspectos significativos de la iniciativa levantada por los gobiernos de Evo Morales y Rafael Correa en el sentido de incorporar la concepción del Buen Vivir como parte de los textos constitucionales y como un esfuerzo válido de transformar estos principios en políticas públicas con todas las dificultades y contradicciones que esto conlleva. Por la misma razón, ellas destacan que dicho esfuerzo se contradice con el modelo neo-extractivista impulsado por ambos gobiernos, modelo que basa su legitimidad en la necesidad imperiosa de generar divisas para financiar los programas sociales y las políticas públicas asistencialistas implementadas por los gobiernos de Bolivia y Ecuador. Para concluir, Fuscaldo y Urquidi plantean - inspirándose enBoaventura de Sousa Santos - que un proyecto a través del cual se orienten las prácticas transformadoras contra-hegemónicas debe forzosamente estar asentado en la desmercantilización - junto con la descolonización y la democratización- de la naturaleza y de las relaciones sociales. En este sentido, el Buen Vivir desde una perspectiva indígena y comunitaria, representa una enorme e indispensable contribución.

Por su parte, en el escrito de Juan Jaime Loera que lleva por título "La construcción de los buenos vivires; entre los márgenes y tensiones ontológicas”, el autor se plantea establecer un debate a partir de las fronte- 
ras que existen entre los diversos tipos de saberes y en los cuales se articulan los proyectos relacionados al Buen Vivir. Con esta finalidad, Loera comienza por resaltar la necesidad de reconocer la pluralidad de significados, discursos y proyectos relacionados al Buen Vivir que están en continua construcción y surgen en diversidad de contextos. Para lograr lo anterior se requiere de un diálogo y un entretejido de las políticas que permitan establecer puentes comunicativos entre los diversos saberes, que expresan la dualidad entre el derecho de vivir de forma diferente a la sociedad dominante y, al mismo tiempo, pertenecer a ella. Según él, la tensión creada entre ambas fuerzas es fundamental para entender el Buen Vivir en el contexto político Latinoamericano contemporáneo. Apoyándose en los casos del pueblo Rarámuri del Norte de México y en las comunidades Mapuche de Chile, el autor observa que ambos pueblos ocupan un doble espacio, demandando por una parte derechos igualitarios y ciudadanía al interior del Estado nacional y del mercado y, por otra parte, ubicándose fuera de dichos espacios como una manera de fortalecer sus capacidades de autogestión y de posicionarsecon proyectos políticos alternativos. Es entonces en este balance de fuerzas, en esta tensión no ausente de conflictos y contradicciones, donde cobra sentido conceptualizar los proyectos del Buen Vivir.

En el sexto artículo de la serie "Principios orientadores en la intervención psicosocial y comunitaria centrada en infancia, interculturalidad y Buen Vivir”, Leyla Méndez y Pablo Rojas exponen los resultados de una investigación-acción-participativaa partir del análisis de una experiencia de intervención psicosocial y comunitaria realizada en un espacio de educación no formal llamado Escuelita intercultural para el Buen Vivir. Por lo mismo, este trabajo no solamente contempla el producto de una investigación en su sentido tradicional, sino que ella se sustenta en un proceso dinámico de fortalecimiento de los vínculos comunitarios por medio de la intervención psicosocial. En dicho contexto, los preceptos del Buen Vivir se articulan con otros principios orientadores claves como praxis crítica, perspectiva situada, perspectiva gestáltica, participación infantil, interculturalidad crítica y perspectiva descolonizadora. De la conjunción entre ellos surge una posibilidad de pensar el Buen Vivir como una alternativa viable para desarrollar y consolidar los saberes de niños y niñas en un contexto comunitario, superando en los hechos aquellas prácticas colonizadoras y paternalistas presentes en los tipos de aprendizaje tradicional.

A su vez Víctor Molina en “Existencia equilibrada. Metáfora del Buen Vivir de los pueblos indígenas" comparte los resultados de una investigación realizada entre el pueblo indígena Nasa de Colombia en la cual se puede apreciar que dicha comunidad valoriza preferencialmente la relación entre los seres humanos y entre todos los seres que forman parte del mundo de la vida, en completa armonía con la Madre Naturaleza. En este agrupamiento se encontró que se encontró que las categorías: existencia equilibrada, territorio y economía de la reciprocidad funcionan como un eje integrador de las formas de vida de la colectividad. Confrontando la noción clásica de desarrollo impuesta por occidente, las comunidades Nasa llevan una existencia equilibrada que implica la convivencia y el respeto de todos 
a partir de un diálogo fluido y consciente del ser humano con los demás seres para construir una vida digna para todos. Por lo mismo, resalta Molina, desde la perspectiva del Buen Vivir, la visión y praxis cotidiana de estas comunidades representa una posibilidad efectiva de construir colectivamente una nueva forma de organización de la vida.

En el octavo texto de este dossier, un grupo de investigadores de la Universidad de La Frontera, sede Temuco, presentan los resultados de su estudio "Mediciones del desarrollo y cultura: el caso del Índice de Desarrollo Humano y la población mapuche en Chile. Avances en torno a conceptos, metodología e evidencia empírica incorporando la noción de Küme Mongen”, en el cual se recogen los principales resultados en torno a una actualización del Índice de Desarrollo Humano aplicado por el PNUD a comunidades Mapuche que habitan en la región de La Araucanía. Para ello, junto con analizar detalladamente los resultados de dicho estudio,los autores proponen incorporar en futuras mediciones aquellos aspectos socioculturales que definen las formas de vida del pueblo mapuche y que se derivan de la noción de Buen Vivir o Küme Mogñen en lengua mapudungun.

A seguir, en el trabajo titulado "A produção da vida material e imaterial em comunidades chiquitanas e quilombolas em Mato Grosso: uma nova/velha forma de existencia”, tres académicos de la Universidad Federal de Mato Grosso exponen los resultados de un estudio realizado en esas dos comunidades tradicionales, una de raíz indígena (Chiquitanos) y la otra un remaneciente de comunidades quilombolas (Capão Verde).En ellas se puede apreciar la existencia de una cultura distinta de aquella que impera en la sociedad capitalista, la cual se caracteriza por la presencia de rasgos de solidaridad y equidad entre sus pares. Para los autores estas dos comunidades y/o pueblos tradicionales poseen especificidades -en sus saberes ancestrales y en sus formas de organización y distribución del trabajo -, que permiten pensar en la emergencia de otro tipo de vida o de alternativas concretas al proyecto hegemónico de la civilización del capital. En este sentido, la producción de vida y la existencia de las comunidades quilombola y chiquitana se proyectan como una posibilidad real de resistencia y emancipación frente a un mundo que se muestra tan contradictorio y desigual.

En la penúltima comunicación de la serie "El movimiento de ecoaldeas como experiencia alternativa de Buen Vivir”, Leonardo Salamanca y Diego Silva se abocan a mostrar como la creación de ecoaldeas en diversos lugares del mundo puede representar un camino viable para concretizar los principios del Buen Vivir. Para ello los autores realizan una breve exposición sobre la trayectoria histórica de las ecoaldeas, apuntando a seguir, los tres principios y prácticas fundamentales en los que se asienta dicho movimiento: recampesinización, pluriactividad y carácter holístico. Por último los autores advierten que a pesar de que muchas de estas experiencias asumen un lenguaje y prácticas que continúan siendo capitalistas (ecoturismo, ecoconstrucción, terapia espiritual de alto costo, etc.), las ecoaldeas son todavía interesantes laboratorios en pequeña escala de una nueva modali- 
dad de convivencia, "nuevas praxis sustentables, coherentes, responsables éticamente, micropolíticamente participativas y con un sentido de respeto profundo hacia la vida humana y natural”.

Finalizando la sección monográfica, el investigador de la Universidad Católica del Maule, sede Talca, Julien Vanhulst nos entrega su colaboración "El laberinto de los discursos del Buen vivir: entre Sumak Kawsay y Socialismo del siglo XXI", en la cual se dedica a analizar los diferentes discursos que se han ido construyendo en torno al concepto Buen Vivir, distinguiendo entre las corrientes indigenitas, socialista y postestructuralista. Estos discursos asociados al Buen Vivir poseen en común la emergencia de una visión crítica y contestadora de los límites alcanzados por las visiones eurocéntricas del desarrollo y del progreso, que inclusive han contaminado algunas de las propuestas discursivas del desarrollo sostenible. Por otro lado, en algunos casos dichos discursos planteanuna contradicción fragrante entre la dimensión normativa y las prácticas concretas asociadas al neo-extractivismo, especialmente en países como Ecuador y Bolivia, lo cual -en opinión del autor- desacredita la capacidad de los gobiernos y los movimientos sociales de impugnar efectivamente la omnipotencia de los mercados y sus bases neoliberales. Este es probablemente el principal desafío que deben asumir los gobiernos progresistas de la región.

El presente número 40 de Polis Revista Latinoamericana -que cumple trece años de trayectoria ininterrumpida-se completa con la sección Propuestas y avances de investigación, la cual incluye un total de once artículos, para finalizar la actual edición con el apartado Comentarios y reseñas de libros en donde se incluyen cinco textos, dos de los cuales abordan publicaciones recientes sobre el Buen Vivir. 


\section{Notas}

${ }^{1}$ Por ejemplo, se calcula que si el consumo medio de energía de Estados Unidos fuese generalizado para el conjunto de la población mundial, las reservas conocidas de petróleo se agotarían en sólo 19 días. Junto con ello, si todos fuésemos tan contaminadores como los ciudadanos medios de Estados Unidos y Canadá, necesitaríamos de nueve planetas Tierra para absorber las emisiones generadas. Ver:Raj Patel, O valor de nada, Rio de Janeiro: Zahar, 2010.

2 Arturo Escobar, Una minga para el postdesarrollo, Lima: Universidad Nacional Mayor de San Marcos, 2010.

${ }^{3}$ En la primera categoría se encuentran las necesidades de Ser, Tener, Hacer y Estar. Y en la segunda categoría se encuentran las necesidades de Subsistencia, Protección, Afecto, Entendimiento, Participación y Ocio. Ver Manfred Max-Neef, Antonio Elizalde y Martín Hopenhayn, Desarrollo a Escala Humana: Una opción para el futuro, Uppsala: CEPAUR/Fundación Dag Hammrskjöld, 1986.

${ }^{4}$ Jacques Godbout y Alain Caillé, L'esprit du don, Paris: Éditions La Decouverte, 1992.

${ }^{5}$ Serge Latouche, Pequeno tratado do decrescimento sereno; tradução Claudia Berliner. São Paulo: Editora Martins Fontes, 2009.

${ }^{6}$ Carl Honoré, Elogio de la lentitud. Un movimiento mundial desafía el culto a la velocidad. Madrid: RBA Libros, 2009.

7 Joel Kovel y Michael Löwy, Manifiesto Ecosocialista, en Revista Capitalism, Nature, Socialism, vol 13, marzo 2002. 